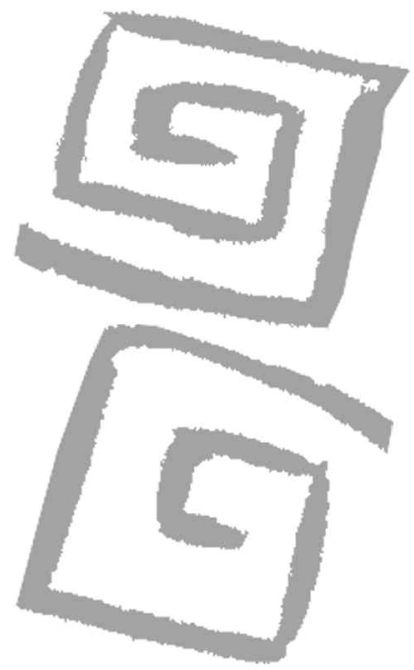

\title{
Evaluación de políticas públicas de provisión de fármacos para diabetes mellitus tipo 2 en Argentina: estudio de caso
}

\author{
Evaluation of public drug provision policies for type 2 \\ diabetes mellitus in Argentina: a case study
}

Elorza, María Eugenia'; Moscoso, Nebel Silvana²; Ripari, Nadia Vanina ${ }^{3}$

${ }^{1}$ Licenciada en Economía. Becaria doctoral del Consejo Nacional de Investigaciones Científicas y Técnicas (CONICET). Ayudante de Cátedra de Estadística, Departamento de Matemática, Universidad Nacional del Sur, Argentina. meugilorz@hotmail.com

2Licenciada en Economía. Doctora en Economía. Investigadora Asistente del Consejo Nacional de Investigaciones Científicas y Técnicas (CONICET). Profesora Adjunta de Economía de la Salud, Departamento de Economía, Universidad Nacional del

Sur, Argentina.

nmoscoso1@gmail.com

3 Licenciada en Economía. Becaria doctoral del Consejo Nacional de Investigaciones Científicas y Técnicas (CONICET). Ayudante de Cátedra de Investigaciones en Salud. Departamento de Ciencias de la Salud, Universidad Nacional del Sur, Argentina.

n_ripari@yahoo.com.ar
RESUMEN En Argentina, la provisión de fármacos para pacientes con diabetes mellitus tipo 2 (DM2) sin cobertura de salud se efectúa mediante programas públicos. En la provincia de Buenos Aires coexisten el programa Remediar, de nivel nacional, y el Programa de Prevención, Diagnóstico y Tratamiento del Paciente Diabético (PRODIABA), de nivel provincial. El presente trabajo estima el porcentaje de población adulta con DM2 sin cobertura del municipio de Bahía Blanca (provincia de Buenos Aires) con necesidad de tratamientos de antidiabéticos orales, que satisface su demanda en el sector público. Es un estudio cuantitativo que evalúa la demanda y la necesidad de provisión pública. Los resultados indican que: 1) el mayor porcentaje de la demanda se satisface en el primer nivel de atención; 2) la provincia de Buenos Aires financia el mayor porcentaje de comprimidos, seguida por el nivel municipal y el nacional; 3) el nivel local también interviene para satisfacer la demanda y 4) la provisión pública total solo daría cobertura a aproximadamente el 25\% de las necesidades en el escenario correspondiente al consumo medio. Esto muestra que, incluso con diferentes programas públicos, la provisión no es suficiente y se requiere de la intervención local aun cuando la descentralización en la adquisición de fármacos no es recomendada por la teoría económica.

PALABRAS CLAVE Diabetes Mellitus; Políticas Públicas; Descentralización; Necesidades y Demanda de Servicios de Salud; Argentina.

ABSTRACT In Argentina, the provision of drugs for patients suffering from type 2 diabetes mellitus who lack health insurance is carried out through public programs. In the Province of Buenos Aires, the national program Remediar and the provincial program PRODIABA (from the Spanish Programa de Prevención, Diagnóstico y Tratamiento del Paciente Diabético) coexist. This study estimates the percentage of adults in the municipality of Bahia Blanca (Province of Buenos Aires) who suffer from type 2 diabetes mellitus and lack health insurance, thus satisfying their need for oral anti-diabetic treatments within the public sector. It is a quantitative study that assesses the need and demand for public provision. The results indicate that: 1 ) the greatest percentage of demand is satisfied at the primary health care level; 2) the province of Buenos Aires funds the largest share of the pills, followed by the municipal and the national levels; 3 ) the local government intervenes to satisfy the demand and 4) the total public provision covers approximately $25 \%$ of the overall need in relation to the average consumption. This shows that despite the presence of these public programs, the provision is insufficient and thus requires the intervention of the local government even though economic theory does not recommend the decentralization of drug purchases.

KEY WORDS Diabetes Mellitus; Public Policies; Decentralization; Health Services Needs and Demand; Argentina. 


\section{INTRODUCCIÓN}

El sistema de salud argentino se organiza a través de tres sectores: el de la seguridad social, el privado y el público. Este último provee servicios en los tres niveles de gobierno (nacional, provincial y municipal) a través de hospitales y centros de atención primaria de salud. Entre otras cuestiones se encarga de: 1) garantizar la cobertura médicosanitaria a personas de bajos ingresos, sin cobertura de salud y/o con limitaciones de acceso geográficas $y, 2$ ) implementar programas para prevenir y/o controlar ciertas patologías, entre ellas las enfermedades crónicas no transmisibles, principales causas de morbimortalidad mundial (1).

En particular, la diabetes mellitus tipo 2 (DM2) es una patología frecuente que absorbe entre el $80 \%$ y el $90 \%$ de los casos de diabetes (2). Si bien la predisposición genética es determinante (3), la suelen desencadenar factores de riesgo tales como obesidad, sedentarismo y tabaquismo $(4,5)$. Su control se logra con ajustes en los hábitos de vida (6-8) y con tratamientos farmacológicos que, frecuentemente, involucran antidiabéticos orales (ADO) (9) tales como glibenclamida y metformina (10).

La relevancia de esta enfermedad se debe a su alta y creciente prevalencia $(11,12)$ y al elevado número de muertes prematuras que provoca (13). Impone una elevada carga socioeconómica a los sistemas de salud pues, a los importantes costos directos del tratamiento (14), se agregan elevados costos indirectos por la pérdida de capacidad para trabajar (15) y costos intangibles asociados al dolor, estrés y discriminación laboral. A su vez, los costos de cualquier prestación médica son mayores en pacientes diabéticos respecto de los no diabéticos (16).

Diversos estudios han estimado la importancia de los costos directos de la enfermedad. En los países desarrollados se observó que el gasto en insulina y $\mathrm{ADO}$ es bajo con relación al gasto en internaciones (17-20). Sin embargo, para Oliva et al., si al gasto en insulina y ADO se le agrega el gasto realizado en otras drogas, el gasto total en medicamentos supera el gasto de las internaciones (21). Según Barceló et al., en América Latina los tratamientos farmacológicos constituyen el $44 \%$ de los costos directos de la diabetes (22).
Dado que en los países de medianos y bajos ingresos entre el 50\% y el $90 \%$ de la población debe pagar por los medicamentos (23), la provisión gratuita de tratamientos farmacológicos para este tipo de enfermedades constituye un aspecto clave de las políticas públicas (24). Según la Federación Internacional de Diabetes, el $61 \%$ de una muestra amplia de países, que incluía Argentina, ha implementado algún programa nacional de diabetes, que habitualmente contempla la provisión gratuita de medicamentos (25).

Diferentes autores han detectado problemas de acceso a los medicamentos para DM2. Según Mendis et al., la disponibilidad de insulina y algunos ADO es escasa en países de ingresos medios y bajos en los que existe provisión gratuita (26). Reséndez et al. observan que las ineficiencias en la gestión de la provisión de medicamentos afectan la disponibilidad de ADO en algunas ciudades de México (27). Viera Paniz et al. advierten la escasez de medicamentos para diabetes en algunas ciudades de Brasil, aún con políticas públicas en los distintos niveles de gobierno (28).

Si bien numerosos estudios analizan las políticas públicas de provisión de fármacos a pacientes con DM2, cobra importancia evaluar estas políticas desde un enfoque de necesidaddemanda-oferta dado que no siempre las necesidades estimadas de una población coinciden con la demanda real de los fármacos bajo estudio (29).

Para estimar las necesidades de bienes o servicios de salud puede utilizarse la información epidemiológica de esa patología, la percepción del individuo o ciertas mediciones clínicas objetivas (30). La demanda estará estrictamente relacionada con los patrones de comportamiento del consumo de los bienes y servicios bajo análisis (31). Existen distintos factores por los que la demanda puede no coincidir con la necesidad, en particular, la presencia de morbilidad no declarada o de enfermedad silenciosa, son algunos de ellos (32).

Desde la teoría económica, la provisión pública de fármacos debe analizarse como un bien privado suministrado públicamente, cuya adquisición justifica políticas con cierto grado de centralización debido a la presencia de economías de escala (33). Sin embargo, las políticas preventivas deben tratarse como bienes públicos cuya 
provisión puede analizarse desde la teoría del federalismo fiscal (34). Bajo este enfoque, la descentralización en la asignación de bienes públicos a los niveles inferiores de gobierno se justifica por las ganancias de bienestar derivadas de adecuar la oferta a las preferencias locales $(35,36)$.

La evidencia latinoamericana revela una gran cantidad de iniciativas locales en materia de política social financiadas en muchos casos con recursos propios (37) que, según Guillen, responden a un proceso especial de descentralización donde los gobiernos locales deciden intervenir en cuestiones a las que no están obligados por el marco institucional (38). Algunos estudios muestran que los niveles locales son fundamentales en la provisión de una amplia variedad de servicios de salud (39) ya que obtienen reducciones en la tasa de mortalidad infantil (40).

Sin embargo, más allá del nivel de gobierno que provea el bien o servicio de salud, es esencial la coordinación entre niveles para garantizar el impacto, la eficacia y la eficiencia de sus políticas públicas (41). En este sentido, Peters sugiere que los problemas de coordinación pueden justificar políticas centralizadas, aunque se pierdan los beneficios de las políticas locales (42). En el caso de las políticas de provisión de medicamentos latinoamericanas, si bien se verificaron amplios beneficios monetarios por las compras centralizadas (43) también se registraron grandes pérdidas asociadas al derroche, robos y/o vencimientos por la distribución a cargo del nivel central (44). En esta región la evidencia sugiere que predominan escasos mecanismos de coordinación entre niveles de gobierno (45) ocasionando dispersión en los efectos de las políticas públicas sociales que persiguen los mismos objetivos (a) (37).

En Argentina, a nivel nacional, la Ley 23.753 de 1989 establece que la nación debe coordinar las políticas que garanticen el acceso a la medicación y que las provincias deben garantizar gratuitamente los tratamientos farmacológicos a los pacientes sin cobertura médica y/o sin recursos (46)

En particular, en la provincia de Buenos Aires, la Ley 11.620 de 1994, propone mejorar la calidad de vida de los pacientes diabéticos (47). En base a la misma se implementa en 1996 el Programa Provincial de Prevención, Diagnóstico y
Tratamiento del Paciente Diabético (PRODIABA), que incluye la provisión gratuita de fármacos a pacientes sin cobertura y/o sin recursos.

A su vez, en el contexto de la crisis socioeconómica de 2001, el Ministerio de Salud de la Nación, con apoyo de organismos internacionales de crédito, implementa el programa nacional Remediar de provisión pública de medicamentos, que incluye medicamentos esenciales, entre ellos, algunos tratamientos para la DM2.

Diferentes autores evaluaron en la provincia de Buenos Aires el acceso a los tratamientos farmacológicos provistos por estos programas. Marín et al., a través de un estudio epidemiológico, estimaron que el programa Remediar en 2004 solo proveía tratamiento continuo anual al 0,65\% de los potenciales pacientes con DM2 (48). A su vez, para Doménech, en el mismo año el PRODIABA daría cobertura al $43 \%$ de la población objetivo (49).

Además de los programas mencionados, en el municipio de Bahía Blanca (provincia de Buenos Aires) se agrega una iniciativa local a través del Programa Municipal de Medicamentos para Patologías Crónicas Prevalentes (en adelante Programa Municipal) que involucra la provisión gratuita de ADO para pacientes con DM2 sin cobertura de salud. Por ello, el presente trabajo se propone analizar en dicho municipio qué porcentaje de población adulta con DM2 sin cobertura de salud con necesidad de tratamientos gratuitos de ADO ha satisfecho su demanda de medicamentos durante el periodo comprendido entre octubre de 2008 y septiembre de 2009.

\section{MATERIAL Y MÉTODOS}

Mediante un estudio descriptivo y cuantitativo se estimaron los tratamientos de ADO efectivamente demandados en el sector público. Dado que los tratamientos demandados públicamente no siempre coinciden con los tratamientos necesarios, se estimó qué porcentaje de la población con necesidades de provisión pública efectivamente recibió el tratamiento. Es un estudio retrospectivo dado que el análisis se realiza desde octubre de 2008 a septiembre de 2009. 
Los tratamientos considerados para el control de la DM2 son: metformina (de 500 mg) y glibenclamida (de $5 \mathrm{mg}$ ).

La metodología implementada para estimar la población adulta con DM2 sin cobertura de salud en el municipio de Bahía Blanca consistió en los siguientes pasos:

1. Se consideró la población adulta (entre 15 y 65 años) de Bahía Blanca en 2008, según las proyecciones estadísticas del Censo Nacional de Población 2001 del Instituto Nacional de Estadísticas y Censos (50).

2. Se estimó la población adulta de Bahía Blanca con necesidad de medicación para DM2, a partir de la tasa de prevalencia regional de diabetes de la región central de Argentina, estimada en un $7 \%$ (51), y de la frecuencia de casos de DM2 en el total de casos de diabetes, estimada en un $0,85 \%$ (2).

3. De la población estimada en el punto anterior se calculó que el 28\% depende de la provisión pública de tratamientos. Esta estimación surge del porcentaje de población sin cobertura de salud estimada para la Región Pampeana en 2005 de acuerdo a la Encuesta Nacional de Factores de Riesgo (52).

Para estimar la demanda efectiva de tratamientos gratuitos de glibenclamida y metformina en el mismo municipio se procedió a:

1. Determinar la provisión gratuita de comprimidos de glibenclamida y metformina para la población adulta con DM2 de Bahía Blanca. Esta información se obtuvo a partir de los datos provistos por organismos de diferentes niveles de gobierno: la Gerencia de Planificación de Remediar+Redes (nivel nacional), los referentes del programa PRODIABA de la Región Sanitaria I (nivel provincial), la Unidad de Diabetes del Servicio de Endocrinología del Hospital Interzonal General de Agudos "Dr. José Penna" (nivel provincial) y la Subdirección de Gestión y Programas de la Secretaría de Salud del Municipio de Bahía Blanca (nivel municipal).

2. Estimar la cantidad de tratamientos provistos públicamente (en monodroga) según tipo de droga y dosis de prescripción. Para ello se consideraron dos tipos de dosis que representan un escenario de mínima y máxima prescripción:

- Dosis media diaria: propuesta para la región por la Asociación Latinoamericana de Diabetes (53), es de $10 \mathrm{mg}$ para la glibenclamida y $1.700 \mathrm{mg}$ para la metformina. Con el propósito de convertir los comprimidos en tratamientos se aplicó para cada fármaco la siguiente fórmula: $N^{\circ}$ dosis medias diarias = unidades disponibles (en mg) por año / dosis media diaria (en mg).

- Dosis máxima diaria: de acuerdo a la teoría indicada por Goodman y Gilman (54) es de 15 mg para la glibenclamida y $2.550 \mathrm{mg}$ para la metformina. En este caso, para convertir los comprimidos en tratamientos se aplicó para cada fármaco la siguiente fórmula: $\mathrm{N}^{0}$ dosis máximas diarias = unidades disponibles (en mg) por año / dosis máxima diaria (en mg).

\section{RESULTADOS}

Según la normativa vigente, los pacientes con DM2 sin cobertura de salud, residentes en el municipio de Bahía Blanca, deberían satisfacer su necesidad de medicación a través de la provisión gratuita del nivel provincial o nacional. Sin embargo, aun reconociendo que la demanda de tratamientos suele ser menor que la necesidad, el nivel local detectó que en el caso de la metformina los tratamientos disponibles no eran suficientes para satisfacer la demanda. Por ello, implementó un programa municipal de provisión gratuita a través del primer nivel de atención para pacientes con patologías crónicas que incluyó la DM2.

En el municipio de Bahía Blanca, la distribución gratuita de los tratamientos de ADO para DM2 se realiza mediante: a) el primer nivel de atención a través de los centros de atención primaria de salud, b) el segundo nivel de atención a través del Hospital Interzonal General de Agudos "Dr. José Penna" y c) la Secretaría de Salud de la Municipalidad de Bahía Blanca.

En el Cuadro 1 se presenta la distribución pública de fármacos según institución, programa y tipo de droga provista. 
Cuadro 1. Distribución pública de medicamentos para DM2 según programa y organismo responsable de su distribución. Bahía Blanca (Argentina). 2008-2009.

\begin{tabular}{lll}
\hline Programa & Organismo responsable de la distribución & Medicamentos \\
\hline PRODIABA & $\begin{array}{l}\text { Hospital Interzonal General de Agudos } \\
\text { "Dr. José Penna"* }\end{array}$ & $\begin{array}{l}\text { Insulina, Glibenclamida, } \\
\text { Metformina }\end{array}$ \\
PRODIABA & $\begin{array}{l}\text { Hospital Municipal de Agudos "Dr. } \\
\text { Leónidas Lucero"** }\end{array}$ & Insulina \\
PRODIABA & $\begin{array}{l}\text { Secretaría de Salud de la Municipalidad } \\
\text { de Bahía Blanca** }\end{array}$ & Glibenclamida, Metformina \\
Programa Municipal & $\begin{array}{l}\text { Secretaría de Salud de la Municipalidad } \\
\text { de Bahía Blanca** }\end{array}$ & Metformina \\
Remediar & $\begin{array}{l}\text { Centros de atención primaria de la } \\
\text { salud** }\end{array}$ & Glibenclamida, Metformina \\
\hline
\end{tabular}

Fuente: Elaboración propia a partir de información suministrada por funcionarios de la Secretaría de Salud de la Municipalidad de Bahía Blanca.

PRODIABA = Programa de Prevención, Diagnóstico y Tratamiento del Paciente Diabético.

Programa Municipal =Programa Municipal de Medicamentos para Patologías Crónicas Prevalentes.

* Jurisdicción provincial.

**Jurisdicción municipal.

El financiamiento de la provisión pública total de cada ADO según nivel de gobierno revela que el nivel provincial, a través del PRODIABA, financia el $87 \%$ de los comprimidos de glibenclamida y más del $70 \%$ de los comprimidos de metformina. Desde el nivel nacional, Remediar financia el $13 \%$ de los comprimidos de glibenclamida y el $6 \%$ de los comprimidos de metformina. Como se observa en la Figura 1 ambos programas satisfacen la demanda local de glibenclamida, aunque no ocurre lo mismo con la metformina donde el nivel municipal debió financiar el $22 \%$ de la misma (b).

Se observan al menos tres cuestiones relevantes referidas al financiamiento de los comprimidos provistos por el sector público:

1. La baja participación del nivel nacional a través de Remediar. Lo cual es posible explicar porque dicho programa surge en un contexto de emergencia sanitaria con el objetivo de mejorar el acceso a los medicamentos esenciales a través del primer nivel de atención, pero no pretende ser el único proveedor público sino complementarse con otras fuentes de financiamiento. Esto se deriva de la legislación nacional que delega a los niveles provinciales la responsabilidad de garantizar el mayor porcentaje de cobertura gratuita de estos tratamientos.

2. En términos agregados, sobre el total de comprimidos demandados, el nivel provincial financia el $77,2 \%$, el nivel municipal el $14,3 \%$ $y$, por último, el nivel nacional el 8,4\%.

3. Con relación al costo total de la provisión local de metformina, que durante el periodo estudiado fue de $\$ 25.000$ ( $\cup \$$ S 6.313), se observa que esta intervención no resulta técnicamente eficiente dado que el costo unitario promedio pagado por el municipio fue de $\$ 0,21$ (U\$S $0,0530)$ (c), mientras que el costo unitario promedio pagado por el nivel nacional (bajo el programa Remediar) fue de $\$ 0,11$ (U\$S 0,0278) (d).

Finalmente y para abordar el objetivo del trabajo, se estimó la cantidad de pacientes que han satisfecho gratuitamente su demanda anual de tratamientos de glibenclamida o metformina. 
Figura 1. Financiamiento de la provisión pública de comprimidos para DM2 por tipo de droga, Bahía Blanca (Argentina). Octubre 2008 - septiembre 2009.

\section{Comprimidos de mefformina}

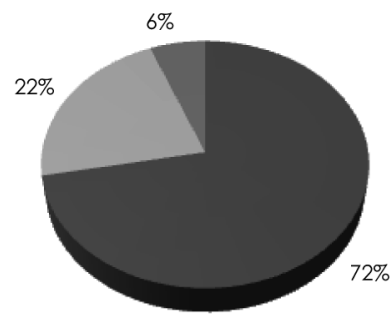

- PRODIABA $\square$ Municipal $=$ Remediar

$n=552.180$

\section{Comprimidos de glibenclamida}

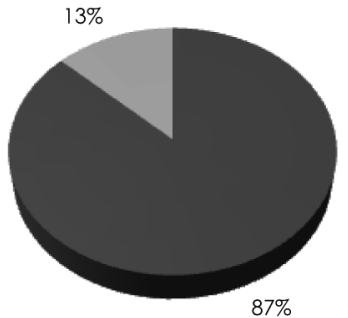

- PRODIABA $=$ Remediar

$n=294.915$

Fuente: Elaboración propia a partir de información suministrada por la Gerencia de Planificación de Remediar+Redes, el Programa de Prevención, Diagnóstico y Tratamiento del Paciente Diabético (PRODIABA), la Unidad de Diabetes del Servicio de Endocrinología del Hospital Interzonal General de Agudos "Dr. José Penna" y la Subdirección de Gestión y Programas de la Secretaría de Salud de Municipio de Bahía Blanca.

Con el propósito de capturar la variabilidad de los tratamientos prescritos entre pacientes se consideraron los escenarios que se corresponden con los diferentes tipos de dosis. Para ello, en el Cuadro 2, se presenta el resultado de convertir la cantidad de comprimidos en cantidades de tratamientos según dosis media diaria y dosis máxima diaria, a partir de las fórmulas mencionadas en "Material y métodos".

Considerando los diferentes escenarios, se observa que los tratamientos anuales provistos gratuitamente a pacientes adultos con DM2 varían entre 301 y 451 en el caso de la metformina y entre 273 y 410 en el caso de la glibenclamida.

A su vez, los tratamientos farmacológicos provistos a través del primer nivel de atención representan el 70\% en el caso de metformina y el $59 \%$ en el caso de la glibenclamida. Estos resultados son acordes a la relevancia que debe tener la atención primaria de la salud en las políticas de prevención, tratamiento y control de esta enfermedad (55).

Cuadro 2. Tratamientos anuales estimados según medicamento y dosis, por programa y nivel de atención. Bahía Blanca (Argentina). Octubre 2008 - septiembre 2009.

\begin{tabular}{|c|c|c|c|c|c|}
\hline \multirow[t]{3}{*}{ Programa } & \multirow[t]{3}{*}{ Nivel de atención } & \multicolumn{4}{|c|}{ Tratamientos anuales } \\
\hline & & \multicolumn{2}{|c|}{ Metformina } & \multicolumn{2}{|c|}{ Glibenclamida } \\
\hline & & $\begin{array}{c}\text { Dosis media } \\
\text { diaria }\end{array}$ & $\begin{array}{c}\text { Dosis máxima } \\
\text { diaria }\end{array}$ & $\begin{array}{c}\text { Dosis media } \\
\text { diaria }\end{array}$ & $\begin{array}{c}\text { Dosis máxima } \\
\text { diaria }\end{array}$ \\
\hline PRODIABA & Primer nivel & 188 & 125 & 190 & 127 \\
\hline Programa Municipal & Primer nivel & 97 & 65 & 0 & 0 \\
\hline Remediar & Primer nivel & 29 & 19 & 53 & 35 \\
\hline PRODIABA & Segundo nivel & 137 & 92 & 167 & 111 \\
\hline \multirow[t]{2}{*}{ Subtotales } & Primer nivel & 314 & 209 & 243 & 162 \\
\hline & Segundo nivel & 137 & 92 & 167 & 111 \\
\hline Totales & & 451 & 301 & 410 & 273 \\
\hline
\end{tabular}

Fuente: Elaboración propia a partir de la información suministrada por la Gerencia de Planificación de Remediar+Redes, el PRODIABA, la Unidad de Diabetes del Servicio de Endocrinología del Hospital Interzonal General de Agudos "Dr. José Penna" y la Subdirección de Gestión y Programas de la Secretaría de Salud del Municipio de Bahía Blanca.

PRODIABA = Programa de Prevención, Diagnóstico y Tratamiento del Paciente Diabético.

Programa Municipal = Programa Municipal de Medicamentos para Patologías Crónicas Prevalentes. 
Una vez estimado el número de pacientes que demandaron tratamientos en el sector público, se estimó la población adulta con DM2 que necesitaría dicha provisión. La relevancia de este análisis radica en que no siempre la necesidad de tratamientos se traduce en demanda efectiva. En nuestro cálculo, la población con necesidades de provisión pública de fármacos sería de 3.274 habitantes. Este valor surge de considerar que en el año 2008 en el municipio de Bahía Blanca habitaban aproximadamente 196.505 personas adultas, de las cuales aproximadamente 13.755 podrían estar enfermas de diabetes de acuerdo a la tasa de prevalencia del $7 \%$ estimada para la región. En particular, 11.691 padecerían DM2. De esta población se calcula que 3.274 habitantes con DM2 no cuentan con cobertura de salud, de acuerdo a la información proporcionada para la región en el año 2005.

Sin embargo, como en toda patología "silenciosa", en diabetes es habitual la presencia de una elevada tasa de subdiagnóstico (es decir, población enferma que aún no ha sido detectada) (56) que Gagliardino et al. han estimado cercana al $50 \%$ en una población de la ciudad de La Plata (Buenos Aires, Argentina) (57).

Si se asume para la ciudad de Bahía Blanca la tasa de subdiagnóstico calculada por Gagliardino et al., la población bajo tratamiento que demanda ADO en el sector público sería aproximadamente la mitad de la población diagnosticada.

Sin embargo, los resultados de la investigación sugieren que la cobertura pública solo sería del $26,30 \%$ de las necesidades, considerando que se consume conforme a la dosis media diaria (861 tratamientos en total, 451 de metformina y 410 de glibenclamida) y del $17,53 \%$ de las necesidades considerando el consumo asociado a la dosis máxima diaria (574 tratamientos, 301 de metformina y 273 de glibenclamida). Estos resultados surgen de estimar que "la población con necesidades de provisión pública de fármacos" es de aproximadamente 3.274 personas.

Es importante aclarar ciertas limitaciones del estudio que podrían distorsionar los resultados obtenidos. Una de ellas es que las dosis utilizadas no consideran a quienes consumen ambas drogas, o tratan su enfermedad únicamente con dieta o con insulina (58).
Otra debilidad está vinculada con la falta de información de dos aspectos claves: 1) el porcentaje de población sin cobertura que adquiere los tratamientos vía gasto de bolsillo, y 2) el porcentaje de tratamientos que es retirado en el municipio de Bahía Blanca por pacientes de la región (esto podría ocurrir principalmente en el hospital provincial).

Las limitaciones mencionadas son factores que podrían determinar que la población efectivamente cubierta por las políticas públicas sea menor o mayor a la estimada. Analizar el impacto y la magnitud de estos factores es la motivación de nuestras futuras líneas de investigación.

\section{DISCUSIÓN}

Los resultados observados en el municipio de Bahía Blanca sugieren que las políticas de distribución gratuita de medicamentos para pacientes de la provincia de Buenos Aires con DM2 tienen fortalezas y debilidades.

Una fortaleza está vinculada con el mecanismo de distribución utilizado, pues la mayor parte de los antidiabéticos orales demandados públicamente han sido entregados a través del primer nivel de atención que, de acuerdo a la evidencia, es el nivel apropiado para abordar la detección, tratamiento y prevención de esta enfermedad.

La principal debilidad es que los tratamientos provistos públicamente por el nivel nacional y provincial en el municipio bajo estudio, no son suficientes para satisfacer la demanda de metformina. Esto motivó la intervención del gobierno local demostrando que el acceso de muchos pacientes diabéticos estuvo garantizado por una decisión voluntaria del nivel más bajo de gobierno y no por los niveles superiores, cuyas leyes los hacen responsables de la provisión de medicamentos y coordinación de las políticas para esta patología.

Estos resultados manifiestan que, en un aspecto clave del sector salud como es la adquisición de medicamentos, intervienen los niveles más bajos de gobierno aun cuando la teoría de la descentralización, basada en el argumento de las economías de escala, no recomienda la intervención local en esta función asignativa. 
Las consecuencias económicas de que los niveles nacional y provincial no satisfagan la demanda y la necesidad de tratamientos son: 1) en lo inmediato, y con respecto a la demanda, la ineficiencia técnica del subsector público, dado que los precios pagados por el nivel local son superiores a los obtenidos por los programas nacionales (en las licitaciones internacionales) o provinciales (con producción de sus propios laboratorios), y 2) en el mediano plazo, los resultados sugieren que aún con políticas públicas implementadas en los tres niveles de gobierno, un bajo porcentaje de los pacientes con necesidades de tratamientos farmacológicos fueron cubiertos desde el sector público. Esto implicará costos directos, indirectos e intangibles asociados a las futuras complicaciones de salud de los pacientes que no acceden en el presente a un tratamiento continuo de la enfermedad.

\section{AGRADECIMIENTOS}

Agradecemos al Lic. Jorge Groppa y al Dr. Gerardo Deblauwe de la Secretaría de Salud de la Municipalidad de Bahía Blanca (Provincia de Buenos Aires, Argentina) quienes suministraron valiosa información para desarrollar este estudio.

\section{NOTAS FINALES}

a. El riesgo de dispersión de esfuerzos de las políticas públicas se analiza a partir de escenarios que determinan cuatro modelos: coordinación jerárquica, dispersión, donante-receptor y gobierno multinivel. En el caso de las políticas sociales latinoamericanas se reconoce la latencia del riesgo de dispersión.

b. Este porcentaje equivale a 119.140 comprimidos y representa más de la mitad de los comprimidos entregados por PRODIABA al primer nivel de atención (230.000). c. Promedio de los precios unitarios (expresados en pesos) pagados en las dos licitaciones públicas municipales realizadas durante el período estudiado, en el que se realizaron compras de metformina. Información proporcionada por la Coordinación de Farmacia dependiente de la Subdirección de Gestión y Programas de la Secretaría de Salud de la Municipalidad de Bahía Blanca.

d. Promedio de los precios unitarios finales pagados en las licitaciones del 2008 y 2009 donde se realizaron compras de metformina. Información proporcionada por la Gerencia de Planificación de Remediar+Redes.

\section{REFERENCIAS BIBLIOGRÁFICAS}

1. World Health Organization. The global burden of disease: 2004 update [Internet]. Geneva: WHO; 2008 [citado 7 feb 2009]. Disponible en: http://www.who.int/healthinfo/global_burden_di sease/2004_report_update/en/index.html

2. Conget I. Diagnóstico, clasificación y patogenia de la diabetes mellitus. Revista Española de Cardiología. 2002; 55(5):528-35.

3. Rich SS. Mapping Genes in Diabetes. Genetic Epidemiological Perspective. Diabetes. 1990; 39(11):1315-1319.
4. Stern MP, González C, Mitchell, BD, Villalpando E, Haffner SM, Hazuda, HP. Genetic and environmental determinants of type II diabetes in Mexico City and San Antonio. Diabetes. 1992;41(4):484-492.

5. Tuomilehto J, Lindström J, Eriksson JG, Valle TT, Hämäläinen $H$, Ilanne-Parikka $P$, et al. Prevention of type 2 diabetes mellitus by changes in lifestyle among subjects with impaired glucose tolerance. New England Journal of Medicine. 2001;344(18):1343-1350.

6. Tuomilehto J, Lindstrom J. The major diabetes prevention trials. Current Diabetes Reports. 2003;3(2):115-122. 
7. Nield L, Moore HJ, Hooper L, Cruickshank JK, Vyas A, Whittaker V, Summerbell CD. Asesoramiento dietético para el tratamiento de la diabetes mellitus tipo 2 en adultos (Revisión Cochrane traducida). La Biblioteca Cochrane Plus [Internet]. 2008 [citado 15 ene 2010];(2). Disponible en: http://www.update-software.com/ pdf/CD004097.pdf

8. Norris SL, Zhang X, Avenell A, Gregg E, Schmid $\mathrm{CH}$, Lau J. Intervenciones no farmacológicas para la pérdida de peso a largo plazo en adultos con prediabetes (Revisión Cochrane traducida). La Biblioteca Cochrane Plus [Internet]. 2008 [citado 28 ene 2010];(2). Disponible en: http://www.update-software.com/pdf/CD005270.pdf

9. Krentz AJ, Bailey CJ. Oral antidiabetic agents: current role in type 2 diabetes mellitus. Drugs. 2005;65(3):385-411.

10. Gagliardino J, Olivera EM, Etchegoyen GS, González C, Guidi ML. Evaluación y costos del proceso de atención de pacientes diabéticos. Medicina (Buenos Aires). 2000;60:880-888.

11. Wild S, Roglic G, Green A, Sicree R, King H. Global prevalence of diabetes: Estimates for the year 2000 and projections for 2030. Diabetes Care. 2004;27(5):1047-1053.

12. Shaw JE, Sicree RA, Zimmet PZ. Global estimates of the prevalence of diabetes for 2010 and 2030. Diabetes Research and Clinical Practice. 2010;87(1):4-14.

13. Roglic G, Unwin N. Mortality attributable to diabetes: estimates for the year 2010. Diabetes Research and Clinical Practice. 2010;87(1):15-19.

14. Zhang $P$, Zhang $X$, Brown J, Vistisen D, Sicree R, Shaw J, et al. Global healthcare expenditure on diabetes for 2010 and 2030. Diabetes Research and Clinical Practice. 2010; 87(3):293-301.

15. American Diabetes Association. Economic costs of diabetes in the U.S. in 2007. Diabetes Care. 2008;31(3):596-615.

16. Rubin RJ, Altman WM, Mendelson DN. Health care expenditures for people with diabetes mellitus, 1992. Journal of Clinical Endocrinology \& Metabolism.1994;78(4):809A-809F.

17. Kangas $T$, Aro $S$, Koivisto VA, Salinto $M$, Laakso M, Reunanen A. Structure cost of health care of diabetic patients in Finland. Diabetes Care. 1996;19(5):494-497.
18. Hart WM, Espinosa C, Rovira J. El coste de la diabetes mellitus conocida en España. Medicina Clínica. 1997;109:289-293.

19. Mata M, Antoñanzas F, Tafalla M, P Sanz P. El coste de la diabetes tipo 2 en España. Gaceta Sanitaria. 2002;16:511-520.

20. Hogan $\mathrm{P}$, Dall $\mathrm{T}$, Nikolov $\mathrm{P}$, American Diabetes Association. Economic costs of diabetes in the U.S. in 2002. Diabetes Care. 2003;26(3):917-932.

21. Oliva J, Lobo F, Molina B, Monereo S. Direct health care costs of diabetic patients in Spain. Diabetes Care. 2004;27(11):2616-2621.

22. Barceló A, Aedo C, Rajpathak S, Robles S. The cost of diabetes in Latin America and the Caribbean. Bulletin of the World Health Organization. 2003;81(1):19-27.

23. Quick JD, Hogerzeil HV, Velasquez G, Rago L. Twenty-five years of essential medicines. Bulletin of the World Health Organization. 2002;80(11):913-914.

24. Tobar F. Políticas para promoción del acceso a medicamentos: El caso del Programa Remediar de Argentina [Internet]. Washington DC: Banco Interamericano de Desarrollo; 2004 [citado 28 may 2009] (Nota técnica de discusión de salud 002/2004). Disponible en: http://www.femeba.org. ar/fundacion/quienessomos/Novedades/remediarbid04.pdf

25. Colagiuri R, Short R, Buckley A. The status of national diabetes programmes: A global survey of IDF member associations. Diabetes Research and Clinical Practice. 2010;87(2):137-142.

26. Mendis S, Fukino K, Cameron A, Laing R, Filipe JA, Khatib $O$, et al. The availability and affordability of selected essential medicines for chronic diseases in six low- and middle-income countries. Bulletin of the World Health Organization. 2007;85(4):279-289.

27. Reséndez C, Garrido F, Gómez-Dante O. Disponibilidad de medicamentos esenciales en unidades de primer nivel de la Secretaría de Salud de Tamaulipas, México. Salud Pública de México. 2000;42(4):298-308.

28. Vieira Paniz VM, Gastal Fassa AC, Facchini LA, Piccini RX, Tomasi E, Thumé E, et al. Free access to hypertension and diabetes medicines among the elderly: a reality yet to be constructed. Cadernos de Saúde Pública. 2010;26(6):11631174. 
29. Desviat $M$, Delgado $M$, González C, Hernández M. Necesidad, demanda y representación social del trastorno mental en el sur de la región de Madrid. Madrid: Instituto Psiquiátrico de Servicios de Salud Mental José Germain; 1993.

30. Salinas MA, Muñoz MF, Barraza de León AR, Villarreal RE, Nuñez RG, Garza EME. Necesidades en salud del diabético usuario del primer nivel de atención. Salud Pública de México. 2001;43(4):324-335.

31. Economics and Development Resource Center, Project Economic Evaluation Division. Analysis of demand and need. En: Handbook for the economic analysis of health sector projects. Philippines: Asian Development Bank; 2000. p. 27-38.

32. Barragán HL. Necesidades, demanda y oferta de atención médica. En: Fundamentos de Salud Pública. La Plata: Editorial de la Universidad Nacional de la Plata; 2007. p. 353-360.

33. Ugalde A, Homedes N. Descentralización del sector salud en América Latina. Gaceta Sanitaria. 2002;16(1):18-29.

34. Moscoso NS, Modarelli R. Descentralización en salud: marco conceptual y políticas públicas en Argentina. Ciencias Económicas. 2009;27(2):155168.

35. Oates WE. Fiscal Federalism. New York: Harcourt Brace Jovanovich; 1972.

36. Oates WE. On the welfare gains from fiscal decentralization. Journal of Public Finance and Public Choice. 1997;2-3:83-92.

37. Cabrero Mendoza E, Zabaleta Solís D. ¿Cómo construir una mística intergubernamental en la política social?: Análisis de cuatro experiencias latinoamericanas. Revista del CLAD Reforma y Democracia [Internet]. 2009 [citado 10 feb 2011]; 43. Disponible en: http://www.clad.org/ portal/publicaciones-del-clad/revista-clad-reforma-democracia/articulos/043-febrero-20091/cabrero

38. Guillén López T. Municipio y política social: experiencias y nuevo paradigma. En: Cabrero Mendoza E, coordinador. Políticas públicas municipales: una agenda en construcción. México DF: Centro de Investigación y Docencia Económicas, Miguel Ángel Porrúa; 2003. p. 289-309.

39. Letelier L. Fiscal decentralization as a mechanism to modernize the State. Journal of Institutional Comparisons. 2004;2(1):15-20.
40. Nana N. Expenditure decentralization and outcomes: Some determinant factors for success from cross country evidence [Internet]. Ethiopia: United Nations, Economic Commission for Africa [citado 10 feb 2011]. Federal Ministry of Finance of Nigeria, The World Bank, OSSAP-MDGs; 2009. Disponible en: http://www.uneca.org/ acgd/events/2009/mdgs-nigeria/docs/Expenditure $\%$ 20decentralization.pdf

41. Franco R. Institucionalidad de las Políticas Sociales: Modificaciones para mejorar su efectividad [Internet]. Chile: Facultad Latinoamericana de Ciencias Sociales; 2004 [citado 20 ene 2011]. Disponible en: http://bibliotecavirtual.clacso.org. ar/ar/libros/chile/flacso/autoridad.pdf

42. Peters BG. The search for coordination and coherence in Public Policy: Return to the center? [Internet] Berlín Conference on the Human Dimensions of Global Environmental Change, 2004 Dic 3-4, Berlín [citado 10 jul 2010]. Disponible en: http://userpage.fu-berlin.de/ffu/ akumwelt/bc2004/download/peters f.pdf

43. Barrillas E. La fragmentación de los sistemas nacionales de salud. Revista Panamericana de Salud Pública. 1997;1(3):246-249.

44. Tobar F. Lecciones aprendidas en la provisión de medicamentos para la atención primaria de la salud. Salud Pública de México. 2008;50(Suppl 4):S463-S469.

45. Jordana J. Relaciones intergubernamentales y descentralización en América Latina: Una perspectiva institucional. Casos de Argentina y Bolivia [Internet]. Washington DC: BID; 2001 [citado 20 feb 2010] (Documento de Trabajo I-22EU). Disponible en: http://cdi.mecon.gov.ar/biblio/ docelec/indes/dt/l-38.pdf

46. Honorable Congreso de la Nación Argentina. Ley 23753, Salud Pública, Diabetes, Programa de Divulgación Sanitaria [Internet]. Buenos Aires: InfoLeg, Ministerio de Economía y Finanzas Públicas; c2005 [citado 20 feb 2010]. Disponible en: http://www.infoleg.gov.ar/infoleglnternet/anexos/0-4999/154/texact.htm

47. Poder Legislativo de la Provincia de Buenos Aires. Ley 11620, Beneficios a los enfermos de diabetes (Provisión de insulina) [Internet]. La Plata: Ministerio de Jefatura de Gabinete de Ministros [citado 20 feb 2010]. Disponible en: http://www.gob.gba.gov.ar/legislacion/legislacion/l-11620.html

48. Marín GH, Canas M, Homar C, Perrotta M. Utilización de medicamentos del Programa 
REMEDIAR en la Provincia de Buenos Aires, Argentina. Latin America Journal of Pharmacy. 2008;27(4):535-542.

49. Doménech M. Programa de prevención de diabetes de la Provincia de Buenos Aires. Boletín PROAPS REMEDIAR [Internet]. 2004 [citado 7 feb 2007];2(9):16-18. Disponible en: http://remediar.gov.ar.pampa.avnam.net/files/Boletin9.PDF

50. Instituto Nacional de Estadística y Censos. Estimaciones de población total por departamento y año calendario: Período 2001-2010 [Internet]. Buenos Aires: INDEC; 2008 [citado 15 may 2010]. (Serie análisis demográfico N³4). Disponible en: http://www.indec.mecon.ar/nuevaweb/cuadros/2/estimaciones-serie34.pdf

51. Sereday MS, Gonzalez C, Giorgini D, De Loredo L, Braguinsky J, Cobeñas C, et al. Prevalence of diabetes, obesity, hypertension and hyperlipidemia in the central area of Argentina. Diabetes \& Metabolism. 2004;30(4):335-339.

52. Ministerio Nacional de Salud- INDEC. Encuesta Nacional de Factores de Riesgo (ENFR) 2005. [citado 20 may 2010]. Disponible en: http://msal.gov.ar/ENT/VIG/Areas_Tematicas/Fact ores_de_Riesgo/PDF/Encuesta $\% 20$ Nacional $\% 20$ de $\% 20$ Factores $\% 20 \mathrm{de} \% 20$ Riesgo $\% 202005$ info rme_final_breve.pdf

53. Asociación Latinoamericana de Diabetes. Guías ALAD 2006 de diagnóstico, control y tratamiento de la Diabetes Mellitus Tipo 2: Tratamiento con antidiabéticos orales. Revista de la Asociación Latinoamericana de Diabetes [Internet]. 2006;14(3):120-128. [citado 25 marzo 2012]. Disponible en: http://revistaalad.com.ar/pdfs/060303cp7.pdf

54. Davis SN, Granner DK. Insulin, oral hypoglucemic agents and pharmecology of the endocrine. En: Hardman JG, Limbird LE, Gilman AG. Goodman \& Gilman's: The pharmacological basis of therapeutics. 10th ed. New York: McGraw-Hill; 2001. p. 1679-1714.

55. Aráuz AG, Sánchez G, Padilla G, Fernández M, Roselló M, Guzmán S. Intervención educativa comunitaria sobre la diabetes en el ámbito de la atención primaria. Revista Panamericana de Salud Pública. 2001;9(3):145-153.

56. Saudek CD, Herman WH, Sacks DB, Bergenstal RM, Edelman D, Davidson MB. A new look at screening and diagnosing diabetes mellitus. Journal of Clinical Endocrinology \& Metabolism. 2008; 93(7):2447-2453.

57. Gagliardino JJ, Olivera EM, Barragán HL, Hernández RE. Diabetes mellitus e hipertensión arterial: aspectos clínicos y epidemiológicos en la población de La Plata. Medicina (Buenos Aires).1995;55:421-430.

58. Gagliardino JJ, De la Hera M, Siri F, Grupo de Investigación Red QUALIDIAB. Evaluación de la calidad de la asistencia al paciente diabético en América Latina. Revista Panamericana de Salud Pública 2001;10(5):309-317.

\section{FORMA DE CITAR}

Elorza ME, Moscoso NS, Ripari NV. Evaluación de políticas públicas de provisión de fármacos para diabetes mellitus tipo 2 en Argentina: estudio de caso. Salud Colectiva. 2012;8(1):35-45.

Recibido el 24 de junio de 2011

Versión final presentada el 19 de octubre de 2011

Aprobado el 14 de noviembre de 2011 